\title{
Testing the hypothesis of accelerated cerebral white matter aging in schizophrenia and major depression
}

\author{
P. Kochunov ${ }^{1,2}$, D.C. Glahn ${ }^{2,3,4}$, L.M. Rowland ${ }^{1}$, R.L. Olvera ${ }^{5}$, A Winkler ${ }^{3}$, Y.H. Yang ${ }^{6}$, H. \\ Sampath $^{1}$, W.T. Carpenter ${ }^{1}$, R. Dugarrila ${ }^{2}$, J. Curran ${ }^{2}$, J. Blangero ${ }^{2}$, and L.E. Hong ${ }^{1}$ \\ ${ }^{1}$ Maryland Psychiatric Research Center, Department of Psychiatry, University of Maryland School \\ of Medicine, Baltimore \\ ${ }^{2}$ Southwest Foundation for Biomedical Research. San Antonio, Texas \\ ${ }^{3}$ Department of Psychiatry, Yale University \& Olin Neuropsychiatric Research Center, \\ Connecticut \\ ${ }^{4}$ Research Imaging Institute, The University of Texas Health Science Center, San Antonio \\ ${ }^{5}$ Department of Psychiatry, The University of Texas Health Science Center, San Antonio \\ ${ }^{6}$ Intramural Research Program, National Institute on Drug Abuse, Baltimore
}

\begin{abstract}
Introduction-Elevated rate of aging-related biological and functional decline, termed accelerated aging, is reported in patients with schizophrenia (SCZ) and major depressive disorder (MDD). We used diffusion tensor imaging (DTI) derived fractional anisotropy (FA) as biomarkers of aging-related decline in white matter (WM) integrity to test the hypotheses of accelerated aging in SCZ and MDD.

Methods-The SCZ cohort was composed of 58/60 SCZ patients/controls (age=20-60years). MDD cohort was composed of 136/351 MDD patients/controls (age=20-79years). Main outcome measures were the diagnosis-by-age interaction on whole-brain-averaged WM FA values and FA values from twelve major WM tracts.
\end{abstract}

\begin{abstract}
Results-Diagnosis-by-age interaction for the whole-brain average FA was significant for the $\mathrm{SCZ}(\mathrm{p}=0.04)$ but not in MDD cohort ( $\mathrm{p}=0.80$ ). Diagnosis-by-age interaction was nominally significant $(\mathrm{p}<0.05)$ for five WM tracts for SCZ and for none of the tracts in the MDD cohort. Tract-specific heterochronicity of the onset of age-related decline in SCZ demonstrated strong negative correlations with the age-of- peak myelination and the rates of age-related decline obtained from normative sample ( $\mathrm{r}=-0.61$ and $-0.80, \mathrm{p}<0.05$, respectively). No such trends existed for MDD cohort.
\end{abstract}

Conclusion-Cerebral WM showed accelerated aging in SCZ but not in MDD, suggesting some difference in the pathophysiology underlying their WM aging changes. Tract-specific

\footnotetext{
() 2012 Society of Biological Psychiatry. Published by Elsevier Inc. All rights reserved.

*Corresponding Author: Maryland Psychiatric Research Center, Department of Psychiatry, University of Maryland School of Medicine, Baltimore, MD, USA, Phone: (410) 402-6110, Fax (410) 502-6778, pkochunov@ mprc.umaryland.edu.

Financial Disclosure

The authors report no biomedical financial intersts or potential conflicts of interest.

Publisher's Disclaimer: This is a PDF file of an unedited manuscript that has been accepted for publication. As a service to our customers we are providing this early version of the manuscript. The manuscript will undergo copyediting, typesetting, and review of the resulting proof before it is published in its final citable form. Please note that during the production process errors may be discovered which could affect the content, and all legal disclaimers that apply to the journal pertain.
} 
heterochronicity of WM development modulated presentation of accelerated aging in SCZ: white matter tracts that matured later in life appeared more sensitive to the pathophysiology of SCZ and demonstrated more susceptibility to disorder-related accelerated decline in FA values with age. This trend was not observed in MDD cohort.

\section{Introduction}

Elevated rate of aging-related clinical, biological and functional decline, termed accelerated aging(1-3), is observed in many clinical diagnoses ranging from progeria and other rare genetic accelerated aging syndromes(3) to more common conditions such as chronic obstructive pulmonary disease(4). Psychiatric disorders such as schizophrenia (SCZ) $(1,4$, $5)$ and major depressive disorder (MDD) $(2,6,7)$ have been postulated to increase individual risks for accelerated aging. Specifically, schizophrenia is associated with increased rate of cardiovascular illnesses and other conditions commonly associated with aging(8). Schizophrenia patients have increased mortality rate and shorter (by as much as twenty years) average lifespan even after accounting for suicide (1,9-11). Similar findings are emerging in $\mathrm{MDD}(2)$. Additionally, patients with these mental disorders are reported to have shortened telomeres, a biomarker potentially associated with accelerated aging $(6,12)$.

This initial evidence for accelerated aging in SCZ and MDD raises an intriguing question on whether the biological processes that lead to accelerated aging are the same in these two diagnostically separated illnesses. This question is best approached using a brain measure(s) that is sensitive for both disorders and simultaneously is a sensitive biomarker for cerebral aging. One such a measure is the fractional anisotropy (FA) of water diffusion which reflects the integrity of the cerebral white matter (WM)(13-16). The FA values are sensitive to agerelated alterations in cerebral $\mathrm{WM}(13,17,18)$ and are used as the endophenotypes, sensitive to the pathophysiology of SCZ and MDD disorders(19-21). We hypothesized a diagnosisby-age interaction in the cerebral WM FA values in patients versus normal controls. We tested this hypothesis in two well-characterized populations: a cohort of SCZ patients with matched controls and a cohort of MDD patients compared with non-depression controls (22).

Schizophrenia and depression are both associated with DTI-FA and cerebreal connectivity deficits $(23,24)$. Reduced FA values are consistently reported in chronic SCZ patients(20, 25). Studies on correlations of FA vand age in SCZ have yielded contradictory results. Jones and colleagues showed a positive correlation (26) of FA with age, while others showed a negative correlation $(19,20)$. This difference could potentially be explained by the younger age-range in Jones et al that corresponded to the portion of FA developmental trajectory with age that peaks in the $3^{\text {rd }}$-to- $4^{\text {th }}$ decade of life $(17,18,27)$.

Deficits in WM integrity in MDD are more regionally specific and found in the genu of corpus callosum (GCC), cingulum and thalamic radiation(28-32). These regional FA measurements are associated with genetic risks for MDD (21), particularly the rsik in developing MDD in later life(21). No study, we are aware of, have examined the accelerated age effect on FA in MDD, or compared such effects between two major psychiatric illnesses.

We hypothesized that DTI-FA can serve as a biomarker to test accelerated aging in SCZ and perhaps MDD. Additionally, WM tracts that carry higher level cognitive functions follow a more protracted developmental trajectory than sensory and motor tracts and, therefore may be more vulnerable to the neurodegenerative effects of illnesses ${ }^{(33,34)}$. Therefore, our second hypothesis was that the WM tracts that reach peak development later in life may be more susceptible to the effects of accelerated aging. Specifically, we evaluated if the normative age of peak for FA values and rates of development and senescence for individual 
WM tracts that were previously calculated from large groups of normally aging individuals (age ranged from 11-90)(27), could explain the tract-wise differences in aging trends among SCZ, MDD, and control subjects.

\section{Methods}

Testing of the study's hypotheses were performed in two distinct cohorts, each with own normal control group.

\section{SCZ cohort}

Subjects-A total of 118 ( 19 females, average age $=37.7 \pm 12.0$ years, range $=20-60$ ) individuals participated in the study at the Maryland Psychiatric Research Center (Table 1). Among them 58 ( 7 females, age $=37.1 \pm 12.4$ years) were diagnosed with schizophrenia and 60 (12 females, age $37.5 \pm 11.9$ years) were age- and gender- frequency matched healthy controls (Table 1). Controls were recruited through media advertisements. Additional clinical and epidemiological information is provided in Table 1. There was no significant difference in the body mass index between SCZ patients and controls (BMI=26.9 \pm 5.6 vs. $27.0 \pm 6.0, \mathrm{p}=0.90$, respectively). The exclusion criteria included diagnosis with hypertension, hyperlipedimia, type 2 diabetes, heart disorders, and major neurological event such as stroke or transient ischemic attack.

Clinical Assessment-Patients were those with current Axis I schizophrenia diagnosis. Controls were subjects without Axis I psychiatric diagnosis. Controls and patients could not have current or past Axis I mood disorder diagnoses. Illicit substance and alcohol abuse and dependence were exclusion criteria, enforced using breathalyzer and toxicology screen prior to each imaging session. Except two medication-free participants, schizophrenia patients were on antipsychotic medications: 3 were on first-generation antipsychotics and the rest were on either secondary-generation or combined first- and second-generation antipsychotics.

Diffusion Tensor MR imaging-Imaging data was collected using a Siemens 3T Allegra MRI (Erlangen, Germany) located at the National Institute on Drug Abuse using a quadrature RF head coil. DTI data was collected using a spin-echo, EPI sequence with a spatial resolution of $1.7 \times 1.7 \times 4.0 \mathrm{~mm}$. The sequence parameters were: $T E / T R=87 / 5000 \mathrm{~ms}$, FOV $=200 \mathrm{~mm}$, axial slice orientation with 35 slices and no gaps, twelve isotropically distributed diffusion weighted directions, two diffusion weighting values ( $b=0$ and $1000 \mathrm{~s} /$ $\mathrm{mm}^{2}$ ). Subjects' head movement was minimized by the use of padding, hardened polyurethane foam, and/or bite bar.

\section{MDD cohort}

Subjects-A total of 483 (311 females, age $=41.6 \pm 13.6$ years, range $=20-79$ ) MexicanAmerican individuals, from 71 families, who participated in the Genetics of Brain Structure and Function (GOBS) study. Among them 132 (96 females, age $=42.3 \pm 12.1$ years) were diagnosed with MDD (Table 1). This cohort was further subdivided into 68 (54 females, age $=42.8 \pm 11.9$ years) subjects who experienced a single major depressive episode and 64 (41 females, age $=41.1 \pm 12.0$ years) who experienced two or more, recurrent depressive episodes. The remaining 351 ( 215 females, age $41.5 \pm 13.9$ years) were without Axis I diagnosis and were treated as controls. Additional epidemiological information is provided in Table 1. Patients with MDD, as expected, scored higher on the Beck's Depression Inventory (BDI) compared with the controls $\left(11.3 \pm 9.0\right.$ vs. $\left.5.6 \pm 5.6, \mathrm{p}=10^{-9}\right)$. There was no significant difference in the body mass index between MDD patients and controls (BMI $=31.5 \pm 6.8$ vs. $30.5 \pm 6.5, \mathrm{p}=0.14$, respectively) (Table 1 ). Alcohol and illicit drug abuse 
and dependence were exclusionary. Schizophrenia and Axis I psychiatric disorder other than MDD were excluded.

Clinical Assessment-All GOBS subjects received face-to-face medical and psychiatric interviews in the subject's language of choice (English or Spanish)(35). The MiniInternational Neuropsychiatric Interview (MINI-Plus)(36) is a semi-structured interview to make diagnoses for DSM-IV psychiatric illnesses. Subjects with possible psychopathology were discussed in case conferences and diagnoses were reached by consensus. MDD was defined with DSM-IV criteria as having single versus two or more distinct episodes of depression lasting two or more weeks.

Diffusion Tensor MR imaging-Imaging data were collected using a Siemens 3T Trio scanner located at the Research Imaging Institute, UTHSCSA. The details of imaging protocol are described elsewhere(18). In short, diffusion tensor data were collected using a single-shot, echo-planar, single refocusing spin-echo, T2-weighted sequence with a spatial resolution of $1.7 \times 1.7 \times 3.0 \mathrm{~mm}$. The sequence parameters were:TE/TR=87/8000ms, FOV $=200 \mathrm{~mm}$, axial slice orientation with 50 slices and no gaps, 55 isotropically distributed diffusion weighted directions, two diffusion weighting values $\left(b=0\right.$ and $\left.700 \mathrm{~s} / \mathrm{mm}^{2}\right)$. These parameters were calculated using an optimization technique that maximizes the contrast to noise ratio for FA measurements(37).

Image processing: DTI data for both cohorts was processed using a tract-based spatial statistics (TBSS) method, distributed as a part of FMRIB Software Library (FSL) package (38) as described elsewhere(18). The population-based, 3D, DTI cerebral WM tract atlas developed in John Hopkins University and distributed with the FSL package(39) was used to calculate population average FA values along the spatial course of twelve, major WM tracts (Table 2, Figure 1) as described elsewhere $(27,40)$.

\section{Statistical analysis}

We employed a statistical approach previously used $(17,18)$ to study diagnosis-by-age interaction on the age related FA trends. Given the known nonlinear trajectory of white matter development, quadratic effect was a prori test although linear effect was also reported. The model also included fixed effects of diagnosis, age and gender. The SCZ cohort was a case-control sample composed of independent subjects from independent families. Subjects from the MDD cohort were recruited from large families and ancestry was included as a part of the modeling process. The effects of the familial aggregation in MDD cohort were modeled by partitioning the intersubject variance into fixed (age, disorder, sex and their interactions) and mixed, within-family, effects using general linear mixed effect model. Modeling familial aggregations using the mixed model allows the results of the analyses to be more representative for a sample of independent subjects.

Estimation of effects of age-by-diagnosis interaction-FA values were modeled as both linear and quadratic function of age, gender and diagnosis using GLME model presented in equation 1 and 2 , respectively.

$$
\begin{gathered}
F A_{i, j} \sim \mathrm{A}+\beta_{a g e} A g e_{i, j}+\beta_{d x} d x_{i, j}+\beta_{a g e \cdot d x} a g e_{i, j} \cdot d x_{i, j}+\beta_{s e x} \operatorname{Sex}_{i, j}+\alpha_{i, j} \\
F A_{i, j} \sim \mathrm{A}+\beta_{\text {age } 2} A g e_{i, j}{ }^{2}+\beta_{d x} d x_{i, j}+\beta_{\text {age } 2 \cdot d x} A_{g e_{i, j}}{ }^{2} \cdot d x_{i, j}+\beta_{s e x} \operatorname{Sex}_{i, j}+\alpha_{i, j}
\end{gathered}
$$


Where $F A_{i, j}$ is the FA for the " $\mathrm{j}$ th" subject from the " $\mathrm{i}$ th" family, $\mathrm{dx}_{\mathrm{i}, \mathrm{j}}$ is the diagnosis [ $0=$ normal control, $1=$ disorder ( $\mathrm{SCZ}$ or MDD)], $\mathrm{A}$ is the constant FA term, $\beta$ is the covariate regression coefficient and $\alpha_{i, j}$ is a coefficient that accounts for random effects. This modeling was performed with the $[R]$ package(41) using the linear mixed effects model library and the maximum likelihood estimation algorithm (42). Finally, the difference of aging effects between single vs. recurrent episode MDD were compared by coding 0 for single and 1 for recurrent in $\mathrm{dx}_{\mathrm{i}, \mathrm{j}}$ in equations 1 and 2.

The age-by-diagnosis interactions were quantified for whole-brain average FA values in our primary hypothesis testing.

In order to examine heterochronicity effects (see below), we first performed exploratory tests for regional, tract-wise FA measurements and the threshold for statistical significance of the diagnosis, age and gender model was chosen at $\mathrm{p} \unlhd \mathbf{0 . 0 0 4}$, to correct for multiple $(\mathrm{n}=12)$ comparisons. Nominally significant findings ( $\mathrm{p} \unlhd .05$ ) were deemed as potentially interesting. The significance of the individual beta coefficients was based on $\mathrm{p} \unlhd \mathbf{} \mathbf{0} .05$.

\section{Effects of anatomic heterochronicity of WM development-Different WM tracts} reach maturation peak at different age. This is termed heterochronicity of the WM development $(18,33)$. We used tract-wise differences in normal cerebral development and senescence, taken from a previous study(18), to elucidate inter-tract differences in aging trends between patients and controls. First, a polynomial model described in Eq. 2 was used to remove the effects of normal aging on the whole-brain and regional FA values. The residual values were Z-normalized, by dividing them by the standard deviation calculated from the respective control group for each cohort. To visualize the remaining difference in aging trajectory between patients and controls, we fitted a linear age trend using a least squares line fitting. By design, the normal controls have a zero-slope line and a negative slope for patients would indicate accelerated aging. The intercept of the 2 slopes represents the age-of-onset where aging related decline begins to accelerate in the patients compared with the controls. This X-intercept value is thus the tract-specific age of onset of the accelerated FA decline associated with the disease. We calculated the X-intercept for each of the 12 tracts. We then obtained known tract-specific age of peak of FA value from a normative dataset(27), representing the age each tract reaches the peak of its maturation before FA declines with senescence. By correlating the $\mathrm{X}$-intercept and the normative age of peak FA across tracts, we test our second hypothesis that disorder-related accelerated aging maybe sensitive to the chronological variations in maturation stages in different fiber tracts.

\section{Results}

\section{Diagnosis and diagnosis-by-age effects for whole-brain FA values}

SCZ cohort-Beta coefficients and their significance for age and gender effects for the whole-brain average FA values are shown in Table 3 . The quadratic and linear age models were significant for the whole-brain FA values ( $\mathrm{p}=6.9 \times 10^{-5}$ and $9.2 \times 10^{-5}$, respectively). There were no significant main effects on diagnosis. Instead, quadratic effects of the diagnosis-by-age interaction were significant $\left(\beta_{\mathrm{age}}{ }^{2 *} \mathrm{scz}=-6.9 \pm 3.4 \cdot 10^{-6} ; \mathrm{p}=0.04\right)$ and linear effects of the diagnosis-by-age interaction approached significance $\left(\beta_{\text {age }} * \mathrm{scz}=\right.$ $\left.-4.9 \pm 2.6 \cdot 10^{-4} ; \mathrm{p}=0.06\right)$.

MDD cohort-The quadratic and linear age models were significant for whole-brain average FA but there were no significant effects of the diagnosis. Neither quadratic or linear effects of the diagnosis-by-age interaction was significant for the MDD cohort $\left(\beta_{\mathrm{age}}{ }^{2 * \mathrm{MDD}}=\right.$ $-0.2 \pm 0.2 \cdot 10^{-6}, \mathrm{p}=0.9$; and $\beta_{\mathrm{age}} \mathrm{MDD}=-0.6 \pm 1.9 \cdot 10^{-4}, \mathrm{p}=0.7$, respectively) (Figure 2 ). There 
were no significant differences in either linear or quadratic diagnosis-by-age interaction between single vs. recurrent episode MDD (Table 4).

\section{Diagnosis and diagnosis-by-age effects for regional FA values}

SCZ cohort-Nominally significant effects of diagnosis were observed for SLF and SFO WM tracts ( $\mathrm{p}=0.04$ and 0.03 , respectively) (Supplemental Table S1). Quadratic diagnosisby-age interaction was nominally significant $(\mathrm{p}<0.05)$ for five $\mathrm{WM}$ tracts, including all three partitions of CC (genu, body, and splenium) and the SLF and SFO tracts. The sign and the magnitude of the diagnosis-by-age coefficients indicated that rate of age-related decline in patients was more than twice the aging rate in the controls (Supplemental Table S1). Nominally significant, linear diagnosis-by-age interaction was also observed for the genu of CC, SLF and SFO tracts ( $\mathrm{p}=0.04,0.04$ and 0.02 , respectively).

MDD cohort-The quadratic diagnosis-by-age interaction was not significant for either whole-brain average FA value or any of the tract-wise measurements. The quadratic decline in average FA in MDD patients was slightly (by $4 \%$ ) accelerated compared to the normal controls (Supplemental Table S3). Effects of linear diagnosis-by-age interaction were not significant for any of the twelve tracts (Supplemental Table S3).

Testing the effects of age-of-onset and the duration of the illness (Table 1) independent of subject's age, we found that they were no significant correlation between whole-brain FA value in either cohort (all $r<0.12$, all $\mathrm{p}>0.2$ ).

\section{Heterochronicity of WM development on disorder-related differences in aging}

SCZ cohort-Patients with schizophrenia demonstrated numerically higher age-related decline in FA in all twelve tracts. The age-of-intercept for the average FA was 32.4 years ( $\mathrm{SE}=11.9$ years) (Figure 2). Regionally, the age-of-intercept varied from 27.7 years $(\mathrm{SE}=11.0)$ for the $\mathrm{GCC}$ to 49.9 years $(\mathrm{SE}=16.4)$ for the thalamic radiation (TR) (Supplemental Figure S1). The age-of -intercept was significantly and negatively correlated with the normative age of FA peak $(\mathrm{r}=-0.61, \mathrm{p}=0.04)$ obtained from a large, independent cohort of normal subjects(18) (Figure 4, top). This means that tracts that are normally matured slowly and reach FA peak late are vulnerable to earlier onset of accelerated aging in FA in SCZ. We also obtained the normative rate of age-related decline in FA(18) and found that it is was negatively and robustly correlated with the age of X-intercept (Pearson's r= $-0.80, \mathrm{p}=0.005$; Figure 4 , bottom). This means that tracts that are normally associated with steeper age-related decline in FA also have earlier onset of accelerated aging in FA in SCZ. Thus, heterochronicity of the tract-specific age-related maturation and decline explained $37 \%$ and $64 \%$, respectively, of the variance on onset of accelerated aging in SCZ.

MDD cohort-Patients with MDD demonstrated numerically higher age-related decline in FA for seven tracts, when compared to normal controls (none were statistically significant: genu of CC, body of CC, internal capsule, external capsule, Cingulum, SLF and inferior frontal occipital) (Supplemental Figure S2). The age of intercept varied from -251.5 years ( standard error $(\mathrm{SE})=72.0$ years) for the Sagittal Striatum to 82.1 years $(\mathrm{SE}=21.7)$ for the IC. These values suggest that there is no evidence of tract-specific accelerated aging occurring during the normal life span in MDD. The age-of-intercept in the MDD group was not significantly correlated with the normative age of FA peak or decline across tracts $(-0.04$, and -0.21 , respectively; $p>.20)$. 


\section{Discussion}

We aimed to test if the rates of age-related decline in the integrity of cerebral WM were higher in patients affected with SCZ or MDD than in normal controls. In SCZ patients, agerelated decline in the whole-brain FA values were more than twice that of controls, significant under the quadratic model. In contrast, the effects of accelerated aging in the whole-brain FA values were not statistically significant in the MDD patients. Exploration of regional differences provided a more wide-spread evidence for accelerated aging in SCZ patients, with nominally significant effects of accelerated aging observed in five, associative WM tracts, but none in MDD. (The closest track that approached statistical significance was genu of CC, $\mathrm{p}=0.09$, not significant, in MDD). Therefore, we observed an intriguing diseasespecific pattern of the accelerated aging in the WM integrity that is specifically present in the SCZ patients while evidence of the same effect was much weaker in MDD, suggesting some differences in the biological processes underlying the FA changes in these two disorders.

This observation prompted the examination of whether the tract-specific differences in schizophrenia could be explained by the heterochronicity of normal cerebral WM development. This analysis revealed that the age at which schizophrenia patients began to demonstrate lower FA values, presumed due to accelerated aging, was robustly explained by age at which normal peak myelination is achieved and by rate of normative age-related decline ( $\mathrm{r}=-0.61$ and -0.80 , respectively). The negative sign of the correlation coefficients indicated that WM tracts that reach peak myelination later in life and those that have steeper normal age-related decline suffered from earlier onset of schizophrenia-specific accelerated aging. The same analysis when performed for the MDD cohort yielded no such relationships. This provides the first evidence not only for relatively disease-specific accelerated aging of cerebral WM in SCZ, but also suggests that tracts that follow a prolonged path to maturity are more vulnerable to and show the earliest effect of SCZrelated acceleration in aging.

Overall, our findings of accelerated decline in FA in SCZ were consistent with findings of accelerated aging for several other biological measures previously reported in this disorder $(19,20)$. A report by Friedman and colleagues demonstrated no significant FA deficits in the young (age $=20-25$ ) first episode schizophrenia patients(20). Similar finding were observed in our study: average $\mathrm{FA}=0.60 \pm 0.02$ and $0.59 \pm 0.02$ for patients and controls, respectively, two-tailed t-test $\mathrm{p}=0.4$, in this age range. The age at which schizophrenia subjects began to demonstrate lower average FA values in our group (32.4 \pm 11.9$)$ was similar to that reported by Mori and colleagues (27 years)(19).

The rate of accelerated age-related decline (ratio of the $\beta_{\text {age }}$ coefficients) observed by Mori was also similar to the rate observed in our cohort (3.0 and 2.8, respectively). In contrast to schizophrenia, we found no evidence for accelerated FA decline in MDD patients. This finding may be explained by published literature which reported reduced FA values for MDD patients are localized to specific regions $(28-32,34,43)$.

The biological basis of the accelerated decline in FA values in schizophrenia may reflect different neuropathological phenomena but accelerated decline in axonal myelination and/or glial cell density is considered one of the likely causes (44-46). Accelerated decline in FA values occurred primarily in the associative WM regions such as the genu of CC that have long developmental trajectories. This protracted course may make it more vulnerable to SCZ-related acceleration in aging. Postmortem studies in schizophrenia lend support to this hypothesis by demonstrating a reduced expression of key oligodendrocyte/myelination genes $(47,48)$ and reductions in the density of oligodendrocytes, specifically in the 
associative fiber tracts that connect prefrontal cortices $(49,50)$. In agreement, our data demonstrated that the associative WM tracts showed a progressively younger age at which schizophrenia patients began to differ from normal controls or having the "onset" of accelerated aging. In fact, over $60 \%$ of regional variability in the tract-specific age of onset was explained by the tract-wise normal rates of cerebral decline, making the normative heterochronicity of FA maturation the single most important factor for the variable onset of accelerated aging on white matter integrity across different fiber tracts in schizophrenia. The protracted development of associative WM may render it to higher susceptibility to the neurodegeneration associated with schizophrenia pathology or factors such as medication or disease induced functional impairments.

Another plausible explanation for accelerated aging in cerebral WM is the genotype-by-age interactions that affect cellular aging. For instance, genetic variation in TP53, a gene central to DNA repair, oligodendrocyte senescence and apoptosis(51), is associated with deficits in the white matter integrity and reduced neurochemicals such as $\mathrm{N}$-acetylaspartate in the frontal WM (51). The TP53 gene is also a candidate gene for schizophrenia(52, 53). Future studies may explore whether this and other genetic variances may play a role in the WM accelerated aging in schizophrenia.

This report is the first attempt to carefully evaluate evidence for accelerated aging in two common psychiatric disorders. We attempted to minimize the impact of common age-related metabolic disorders such as hypertension, diabetes, heart disorders and stokes by excluding affected subjects from both cohorts. We cannot rule out effects from chronic antipsychotic exposure in SCZ cohort. The correlation between whole-brain FA and current antipsychotic medication dose, as calculated by chlorpromazine equivalent (CPZ), was not significant $(\mathrm{r}=0.02 ; \mathrm{p}=0.9)$. Similar findings were observed for correlation between $\mathrm{CPZ}$ and regional, by tract, FA measurements (all $\mathrm{r}<=0.15$, all $\mathrm{p}>0.25$ ). This design would not allow differentiation or even speculation if the findings in SCZ cohort are primarily caused by the schizophrenia etiology, or by the effects of chronic antipsychotic medication exposure.

Ascertainment of large antipsychotic-naive SZ patient group across different ages to rule out medication effects is difficult but a recent study in newly diagnosed, antipsychotic-naive men demonstrated lower testosterone level, which was thought to be consistent with accelerated aging hypothesis(54).

Mental disorders are also associated with lower socioeconomic status and exposure to chronic stress, both of which are considered unfavorable mediators of aging(2). These factors could increase the likelihood of physical disease and lead to accelerated aging in SCZ. However, these factors can be the predisposition and/or the consequences of SCZ, making a confirmatory analysis difficult. Nevertheless, lower socioeconomic status and chronic stress are commonly reported in both mental disorders, and yet we did not observe WM accelerated aging in the MDD patients. Recent research identified several cellular biomarkers that may potentially be sensitive to chronic stress exposure $(55,56)$. Further studies will be necessary to evaluate if these biomarkers can predict the WM aging trends in SCZ.

There was a higher incidence of smoking in SCZ (Table 1). Short-term tobacco smoking is associated with elevation in FA $(57,58)$, while long-term smoking leads to decline in FA $(59,60)$. We re-analyzed our data only in non-smoker SCZ vs. non-smoker controls, and found that SCZ patients still experienced higher rates of age-related decline than normal controls $\left(\beta_{\text {age } * \text { scz }}=-0.0015 \pm 0.0006, \mathrm{t}=1.8 ; \mathrm{p}=0.06\right)$. While this is not significant due to small sample, there is a clear trend that is consistent with the finding of accelerated aging of white matter integrity in SCZ. 
Many studies have identified abnormal FA in SCZ and MDD, to the point that they appear all the same. The difference on the age-related decline trend in FA between the two illnesses provides an important insight on the nature of the illness-specific FA changes. Note that our conclusions regarding the diagnostic specificity of accelerated aging are limited by the use of the two cohorts with different backgrounds and somewhat different imaging parameters. However, each disease cohort has own matched controls of the same population background, and we applied the same data analysis approach for both cohorts. These approaches provided assurance that the identified disease-specific accelerated aging cannot be biased by differences in the controls.

This study is limited by the smaller size of the schizophrenia cohort. Nonetheless, the finding was statistically significant and this finding's biological validity was further supported by a strong association with WM heterochronicity, a well-established biological process. This study is also limited by the cross-sectional nature of the data, which could biases the finding due to heterogeneity of individual aging trends. Additionally, a shorter life span of schizophrenia patients compared with the controls may bias the estimate of the accelerated aging, although this bias would likely lead to less differences found. Overall, the aim of the study was to examine whether accelerated aging is present in one or both of the key psychiatric illnesses, for which we found relatively clear evidence of disease-specific accelerated aging in schizophrenia but not in major depressive disorder.

\section{Supplementary Material}

Refer to Web version on PubMed Central for supplementary material.

\section{Acknowledgments}

This research was supported by National Institute of Health grants K01EB006395 and R01EB015611 to P.K., R37MH059490 and R01MH078111 to JB, R01MH0708143 and R01MH083824 to D.G, and R01DA027680 and R01MH085646 to L.E.H, and the National Institute on Drug Abuse Intramural Research Program.

\section{References}

1. Kirkpatrick B, Messias E, Harvey PD, Fernandez-Egea E, Bowie CR. Is schizophrenia a syndrome of accelerated aging? Schizophr Bull. 2008; 34:1024-1032. [PubMed: 18156637]

2. Wolkowitz OM, Reus VI, Mellon SH. Of sound mind and body: depression, disease, and accelerated aging. Dialogues Clin Neurosci. 2011; 13:25-39. [PubMed: 21485744]

3. Capell BC, Collins FS, Nabel EG. Mechanisms of cardiovascular disease in accelerated aging syndromes. Circ Res. 2007; 101:13-26. [PubMed: 17615378]

4. Ito K, Barnes PJ. COPD as a disease of accelerated lung aging. Chest. 2009; 135:173-180. [PubMed: 19136405]

5. Jeste DV, Wolkowitz OM, Palmer BW. Divergent trajectories of physical, cognitive, and psychosocial aging in schizophrenia. Schizophr Bull. 2011; 37:451-455. [PubMed: 21505111]

6. Simon NM, Smoller JW, McNamara KL, Maser RS, Zalta AK, Pollack MH, et al. Telomere shortening and mood disorders: preliminary support for a chronic stress model of accelerated aging. Biol Psychiatry. 2006; 60:432-435. [PubMed: 16581033]

7. Lung FW, Chen NC, Shu BC. Genetic pathway of major depressive disorder in shortening telomeric length. Psychiatr Genet. 2007; 17:195-199. [PubMed: 17417064]

8. Hennekens CH, Hennekens AR, Hollar D, Casey DE. Schizophrenia and increased risks of cardiovascular disease. Am Heart J. 2005; 150:1115-1121. [PubMed: 16338246]

9. Tsuang MT, Woolson RF. Excess mortality in schizophrenia and affective disorders. Do suicides and accidental deaths solely account for this excess? Arch Gen Psychiatry. 1978; 35:1181-1185. [PubMed: 697536] 
10. Brown S. Excess mortality of schizophrenia. A meta-analysis. Br J Psychiatry. 1997; 171:502-508. [PubMed: 9519087]

11. Saha S, Chant D, McGrath J. A systematic review of mortality in schizophrenia: is the differential mortality gap worsening over time? Arch Gen Psychiatry. 2007; 64:1123-1131. [PubMed: 17909124]

12. Fernandez-Egea E, Bernardo M, Heaphy CM, Griffith JK, Parellada E, Esmatjes E, et al. Telomere length and pulse pressure in newly diagnosed, antipsychotic-naive patients with nonaffective psychosis. Schizophr Bull. 2009; 35:437-442. [PubMed: 19279086]

13. Pfefferbaum A, Sullivan EV, Hedehus M, Lim KO, Adalsteinsson E, Moseley M. Age-related decline in brain white matter anisotropy measured with spatially corrected echo-planar diffusion tensor imaging. Magn Reson Med. 2000; 44:259-268. [PubMed: 10918325]

14. Song SK, Sun SW, Ju WK, Lin SJ, Cross AH, Neufeld AH. Diffusion tensor imaging detects and differentiates axon and myelin degeneration in mouse optic nerve after retinal ischemia. Neuroimage. 2003; 20:1714-1722. [PubMed: 14642481]

15. Song SK, Yoshino J, Le TQ, Lin SJ, Sun SW, Cross AH, et al. Demyelination increases radial diffusivity in corpus callosum of mouse brain. Neuroimage. 2005; 26:132-140. [PubMed: 15862213]

16. Kochunov P, Thompson PM, Lancaster JL, Bartzokis G, Smith S, Coyle T, et al. Relationship between white matter fractional anisotropy and other indices of cerebral health in normal aging: tract-based spatial statistics study of aging. Neuroimage. 2007; 35:478-487. [PubMed: 17292629]

17. Hasan KM, Kamali A, Iftikhar A, Kramer LA, Papanicolaou AC, Fletcher JM, et al. Diffusion tensor tractography quantification of the human corpus callosum fiber pathways across the lifespan. Brain Res. 2009; 1249:91-100. [PubMed: 18996095]

18. Kochunov P, Glahn DC, Lancaster J, Thompson PM, Kochunov V, Rogers B, et al. Fractional anisotropy of cerebral white matter and thickness of cortical gray matter across the lifespan. Neuroimage. 2011; 58:41-49. [PubMed: 21640837]

19. Mori T, Ohnishi T, Hashimoto R, Nemoto K, Moriguchi Y, Noguchi H, et al. Progressive changes of white matter integrity in schizophrenia revealed by diffusion tensor imaging. Psychiatry Res. 2007; 154:133-145. [PubMed: 17276660]

20. Friedman JI, Tang C, Carpenter D, Buchsbaum M, Schmeidler J, Flanagan L, et al. Diffusion tensor imaging findings in first-episode and chronic schizophrenia patients. Am J Psychiatry. 2008; 165:1024-1032. [PubMed: 18558643]

21. Glahn DC, Curran JE, Winkler AM, Carless MA, Kent JW Jr, Charlesworth JC, et al. High Dimensional Endophenotype Ranking in the Search for Major Depression Risk Genes. Biol Psychiatry. 2011; 71:6-14. [PubMed: 21982424]

22. Mitchell BD, Kammerer CM, Blangero J, Mahaney MC, Rainwater DL, Dyke B, et al. Genetic and environmental contributions to cardiovascular risk factors in Mexican Americans. The San Antonio Family Heart Study. Circulation. 1996; 94:2159-2170. [PubMed: 8901667]

23. Kanaan RA, Kim JS, Kaufmann WE, Pearlson GD, Barker GJ, McGuire PK. Diffusion tensor imaging in schizophrenia. Biol Psychiatry. 2005; 58:921-929. [PubMed: 16043134]

24. White T, Nelson M, Lim KO. Diffusion tensor imaging in psychiatric disorders. Top Magn Reson Imaging. 2008; 19:97-109. [PubMed: 19363432]

25. Perez-Iglesias R, Tordesillas-Gutierrez D, McGuire PK, Barker GJ, Roiz-Santianez R, Mata I, et al. White matter integrity and cognitive impairment in first-episode psychosis. Am J Psychiatry. 2011; 167:451-458. [PubMed: 20160006]

26. Jones DK, Catani M, Pierpaoli C, Reeves SJ, Shergill SS, O'Sullivan M, et al. Age effects on diffusion tensor magnetic resonance imaging tractography measures of frontal cortex connections in schizophrenia. Hum Brain Mapp. 2006; 27:230-238. [PubMed: 16082656]

27. Kochunov P, Williamson DE, Lancaster J, Fox P, Cornell J, Blangero J, et al. Fractional anisotropy of water diffusion in cerebral white matter across the lifespan. Neurobiol Aging. 2012; 33:9-20. [PubMed: 20122755]

28. Kieseppa T, Eerola M, Mantyla R, Neuvonen T, Poutanen VP, Luoma K, et al. Major depressive disorder and white matter abnormalities: a diffusion tensor imaging study with tract-based spatial statistics. J Affect Disord. 2011; 120:240-244. [PubMed: 19467559] 
29. Zhang A, Leow A, Ajilore O, Lamar M, Yang S, Joseph J, et al. Quantitative Tract-Specific Measures of Uncinate and Cingulum in Major Depression Using Diffusion Tensor Imaging. Neuropsychopharmacology. 2011

30. Korgaonkar MS, Grieve SM, Koslow SH, Gabrieli JD, Gordon E, Williams LM. Loss of white matter integrity in major depressive disorder: Evidence using tract-based spatial statistical analysis of diffusion tensor imaging. Hum Brain Mapp. 2011; 32:2161-2171. [PubMed: 21170955]

31. Blood AJ, Iosifescu DV, Makris N, Perlis RH, Kennedy DN, Dougherty DD, et al. Microstructural abnormalities in subcortical reward circuitry of subjects with major depressive disorder. PLoS One. 2011; 5:e13945. [PubMed: 21124764]

32. Allan CL, Sexton CE, Kalu UG, McDermott LM, Kivimaki M, Singh-Manoux A, et al. Does the Framingham Stroke Risk Profile predict white-matter changes in late-life depression? Int Psychogeriatr. 2011:1-8.

33. Flechsig P. Developmental (myelogenetic) localisation of the cerebral cortex in the human. Lancet. 1901; 158:1027-1030.

34. Kochunov P, Glahn DC, Lancaster J, Winkler A, Karlsgodt K, Olvera RL, et al. Blood pressure and cerebral white matter share common genetic factors in Mexican Americans. Hypertension. 2011; 57:330-335. [PubMed: 21135356]

35. Olvera RL, Bearden CE, Velligan DI, Almasy L, Carless MA, Curran JE, et al. Common genetic influences on depression, alcohol, and substance use disorders in Mexican-American families. Am J Med Genet B Neuropsychiatr Genet. 2010; 156:561-568.

36. Sheehan DV, Lecrubier Y, Sheehan KH, Amorim P, Janavs J, Weiller E, et al. The MiniInternational Neuropsychiatric Interview (M.I.N.I.): the development and validation of a structured diagnostic psychiatric interview for DSM-IV and ICD-10. J Clin Psychiatry. 1998; 59(Suppl 20): 22-33. quiz 34-57. [PubMed: 9881538]

37. Jones DK, Horsfield MA, Simmons A. Optimal strategies for measuring diffusion in anisotropic systems by magnetic resonance imaging. Magn Reson Med. 1999; 42:515-525. [PubMed: 10467296]

38. Smith SM, Jenkinson M, Johansen-Berg H, Rueckert D, Nichols TE, Mackay CE, et al. Tractbased spatial statistics: Voxelwise analysis of multi-subject diffusion data. Neuroimage. 2006; 31:1487-1505. [PubMed: 16624579]

39. Wakana S, Jiang H, Nagae-Poetscher LM, van Zijl PC, Mori S. Fiber tract-based atlas of human white matter anatomy. Radiology. 2004; 230:77-87. [PubMed: 14645885]

40. Kochunov P, Glahn D, Nichols T, Winkler A, Hong E, Holcomb H, et al. Genetic Analysis of Cortical Thickness and Fractional Anisotropy of Water Diffusion in the Brain. Frontiers in Neuroscience. 2011; 5:1-15. [PubMed: 21390287]

41. R-Development-Core-Team. R: A Language and Environment for Statistical Computing. 2009.

42. Pinheiro, J.; Bates, D.; DebRoy, S.; Sarkar, D. nlme: Linear and Nonlinear Mixed Effects Models. 2008.

43. Dalby RB, Frandsen J, Chakravarty MM, Ahdidan J, Sorensen L, Rosenberg R, et al. Depression severity is correlated to the integrity of white matter fiber tracts in late-onset major depression. Psychiatry Res. 2011; 184:38-48. [PubMed: 20832255]

44. Gao W, Lin W, Chen Y, Gerig G, Smith JK, Jewells V, et al. Temporal and spatial development of axonal maturation and myelination of white matter in the developing brain. AJNR Am J Neuroradiol. 2009; 30:290-296. [PubMed: 19001533]

45. Abe O, Aoki S, Hayashi N, Yamada H, Kunimatsu A, Mori H, et al. Normal aging in the central nervous system: quantitative MR diffusion-tensor analysis. Neurobiol Aging. 2002; 23:433-441. [PubMed: 11959406]

46. Roussos P, Katsel P, Davis KL, Bitsios P, Giakoumaki SG, Jogia J, et al. Molecular and genetic evidence for abnormalities in the nodes of ranvier in schizophrenia. Arch Gen Psychiatry. 2012; 69:7-15. [PubMed: 21893642]

47. Davis KL, Stewart DG, Friedman JI, Buchsbaum M, Harvey PD, Hof PR, et al. White matter changes in schizophrenia: evidence for myelin-related dysfunction. Arch Gen Psychiatry. 2003; 60:443-456. [PubMed: 12742865] 
48. Mitkus SN, Hyde TM, Vakkalanka R, Kolachana B, Weinberger DR, Kleinman JE, et al. Expression of oligodendrocyte-associated genes in dorsolateral prefrontal cortex of patients with schizophrenia. Schizophr Res. 2008; 98:129-138. [PubMed: 17964117]

49. Hof PR, Haroutunian V, Friedrich VL Jr, Byne W, Buitron C, Perl DP, et al. Loss and altered spatial distribution of oligodendrocytes in the superior frontal gyrus in schizophrenia. 2003; 53:1075-1085.

50. Uranova NA, Vostrikov VM, Orlovskaya DD, Rachmanova VI. Oligodendroglial density in the prefrontal cortex in schizophrenia and mood disorders: a study from the Stanley Neuropathology Consortium. Schizophr Res. 2004; 67:269-275. [PubMed: 14984887]

51. Molina V, Papiol S, Sanz J, Rosa A, Arias B, Fatjo-Vilas M, et al. Convergent evidence of the contribution of TP53 genetic variation (Pro72Arg) to metabolic activity and white matter volume in the frontal lobe in schizophrenia patients. Neuroimage. 2011; 56:45-51. [PubMed: 21296169]

52. Lung FW, Shu BC, Kao WT, Chen CN, Ku YC, Tzeng DS. Association of DRD4 uVNTR and TP53 codon 72 polymorphisms with schizophrenia: a case-control study. BMC Med Genet. 2009; 10:147. [PubMed: 20040103]

53. Ni X, Trakalo J, Valente J, Azevedo MH, Pato MT, Pato CN, et al. Human p53 tumor suppressor gene (TP53) and schizophrenia: case-control and family studies. Neurosci Lett. 2005; 388:173178. [PubMed: 16039051]

54. Fernandez-Egea E, Garcia-Rizo C, Miller B, Parellada E, Justicia A, Bernardo M, et al. Testosterone in newly diagnosed, antipsychotic-naive men with nonaffective psychosis: a test of the accelerated aging hypothesis. Psychosom Med. 2011; 73:643-647. [PubMed: 21949421]

55. Wolkowitz OM, Mellon SH, Epel ES, Lin J, Dhabhar FS, Su Y, et al. Leukocyte telomere length in major depression: correlations with chronicity, inflammation and oxidative stress--preliminary findings. PLoS One. 2011; 6:e17837. [PubMed: 21448457]

56. Wolkowitz OM, Burke H, Epel ES, Reus VI. Glucocorticoids. Mood, memory, and mechanisms. Ann N Y Acad Sci. 2009; 1179:19-40. [PubMed: 19906230]

57. Hudkins M, O’Neill J, Tobias MC, Bartzokis G, London ED. Cigarette smoking and white matter microstructure. Psychopharmacology (Berl). 2010

58. Liao Y, Tang J, Deng Q, Deng Y, Luo T, Wang X, et al. Bilateral fronto-parietal integrity in young chronic cigarette smokers: a diffusion tensor imaging study. PLoS One. 2011; 6:e26460. [PubMed: 22069452]

59. Gons RA, van Norden AG, de Laat KF, van Oudheusden LJ, van Uden IW, Zwiers MP, et al. Cigarette smoking is associated with reduced microstructural integrity of cerebral white matter. Brain. 2011; 134:2116-2124. [PubMed: 21705426]

60. Kim SH, Yun CH, Lee SY, Choi KH, Kim MB, Park HK. Age-dependent association between cigarette smoking on white matter hyperintensities. Neurol Sci. 2010; 33:45-51. [PubMed: 21562840] 


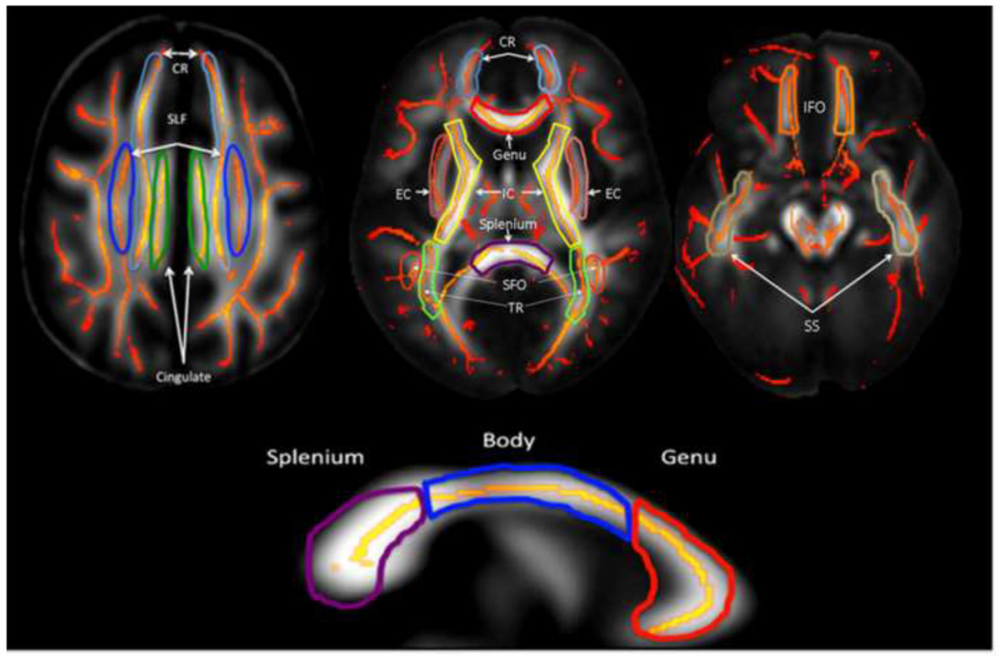

Figure 1.

Average DTI FA values were measured for twelve major cerebral WM tracts. Genu of Corpus Callosum (GCC), Body of Corpus Callosum (BCC), Splenium of Corpus Callosum (SCC), Cingulum (CG), Corona Radiata (CR), External Capsule (EC), Internal Capsule (IC), Superior Frontal-Occipital (SFO), Inferior Frontal Occipital (IFO), Thalamic Radiation (TR), Sagittal Stratum (SS), Superior Longitudinal Fasciculus (SLF). 


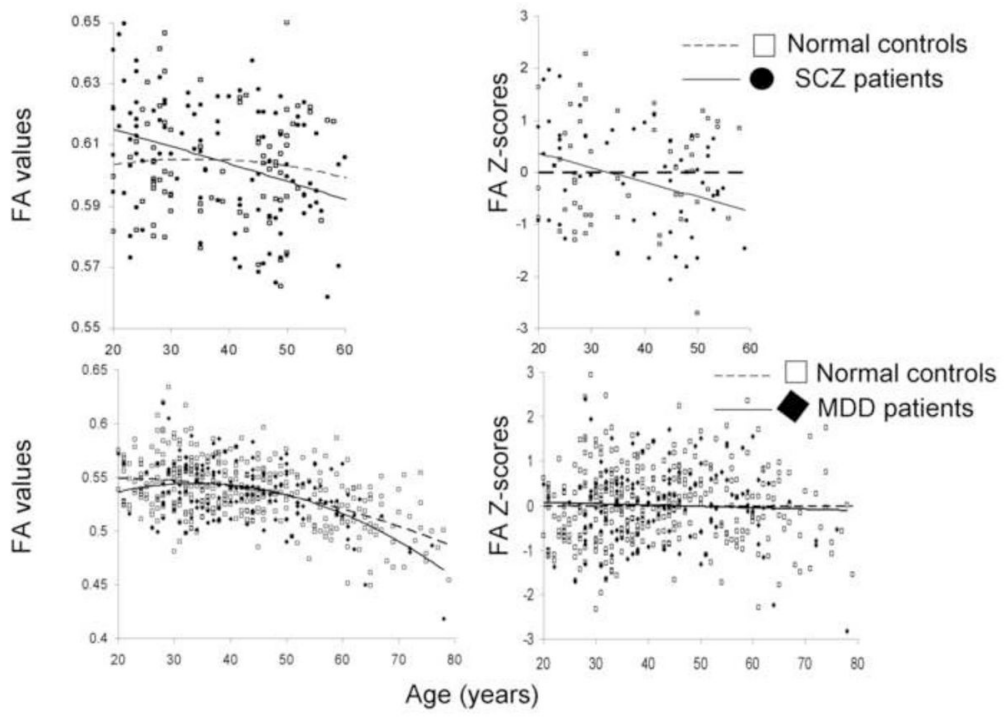

Figure 2.

Whole brain average FA values in schizophrenia patients (top) and major depressive disorder patients (bottom) compared with normal controls. The plots on the left are from the raw FA values. The aging trends were regressed using a polynomial model (Eq. 2) from the raw FA values. The plots on the right are the residual values that were $\mathrm{z}$-normalized so that the regression line across age ( $\mathrm{x}$-axis) was horizontal for the normal controls, for easy visualization. Notable is that the slopes of the decline in FA values with age was significant in schizophrenia $(\mathrm{y}=-0.031 \mathrm{x}+0.96 ; \mathrm{r}=0.32, \mathrm{p}=0.02)$ but not in the MDD $(\mathrm{y}=-0.0008 \mathrm{x}$ $+0.021 ; \mathrm{r}=0.00 ; \mathrm{p}=0.99)$ patients. 


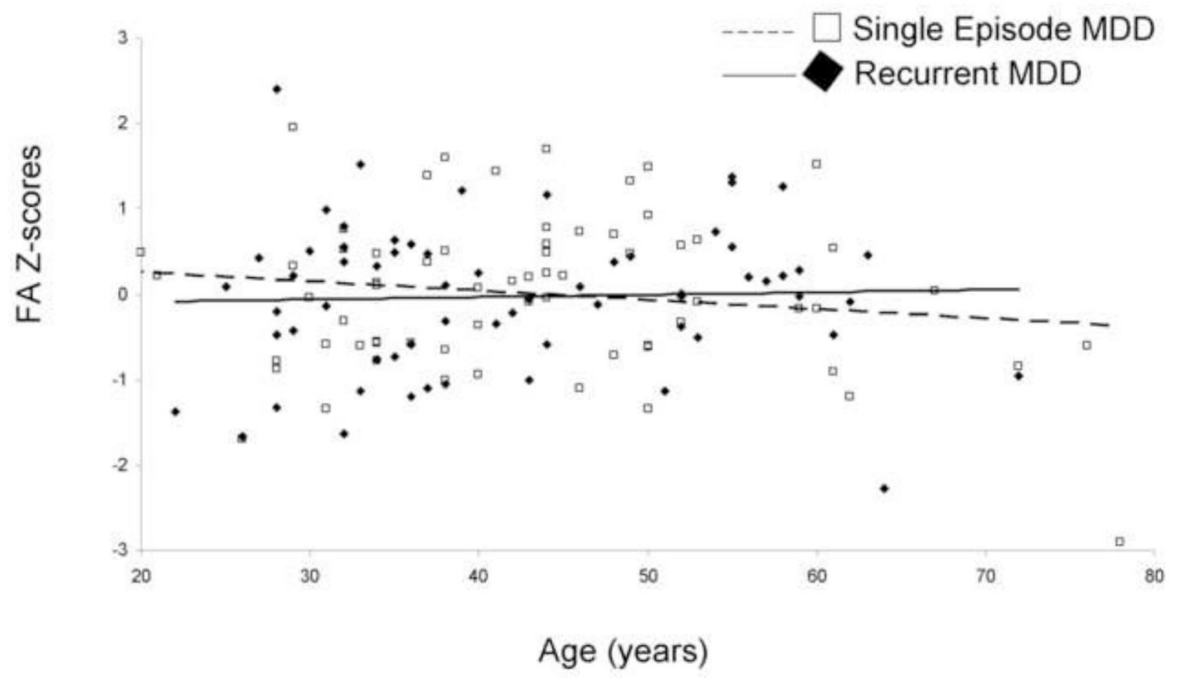

Figure 3.

Whole brain average FA values (z-normalized to normal controls) in single episode and recurrent MDD patients were similar. 


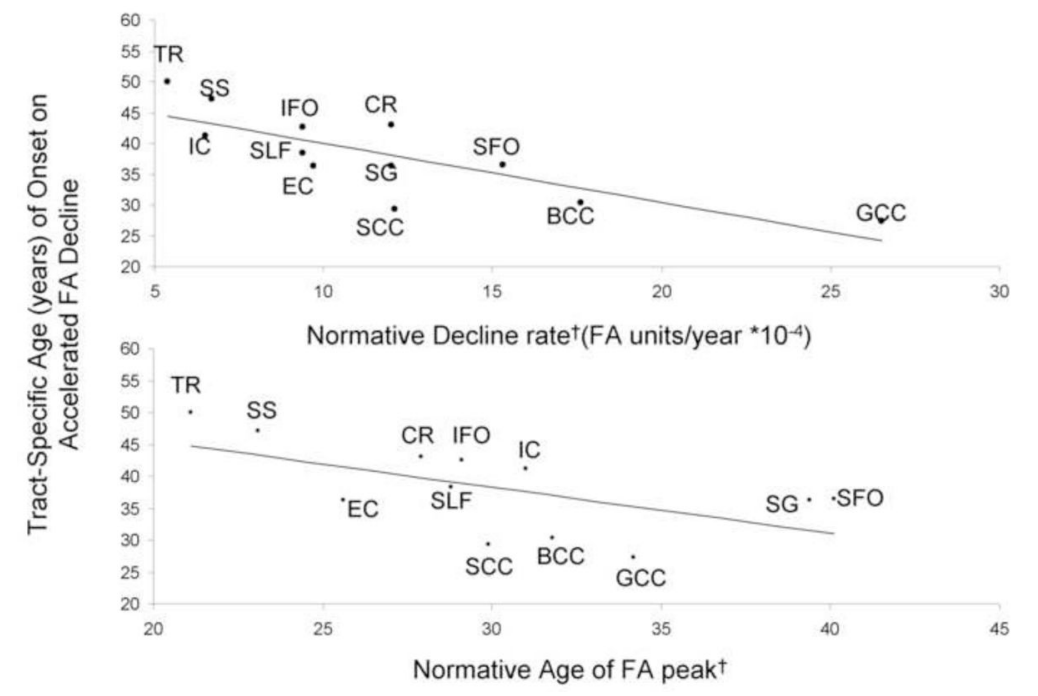

Figure 4.

Age of X-intercept for schizophrenia patients plotted for twelve WM tracts is correlated with normative variations in tract-specific rate of age-related decline $\left(\mathrm{y}=-0.75^{*} \mathrm{x}+60.9 ; \mathrm{r}=-0.81\right)$ (top graph) and age of FA peak $(y=-0.48 * x+48.5 ; r=-0.60)$ (bottom graph). $\dagger$ Normative data is taken from(27), also tabulated in Table 1. Genu of Corpus Callosum (GCC), Body of Corpus Callosum (BCC), Splenium of Corpus Callosum (SCC), Cingulum (CG), Corona Radiata (CR), External Capsule (EC), Internal Capsule (IC), Superior Frontal-Occipital (SFO), Inferior Frontal Occipital (IFO), Thalamic Radiation (TR), Sagittal Stratum (SS), Superior Longitudinal Fasciculus (SLF) 


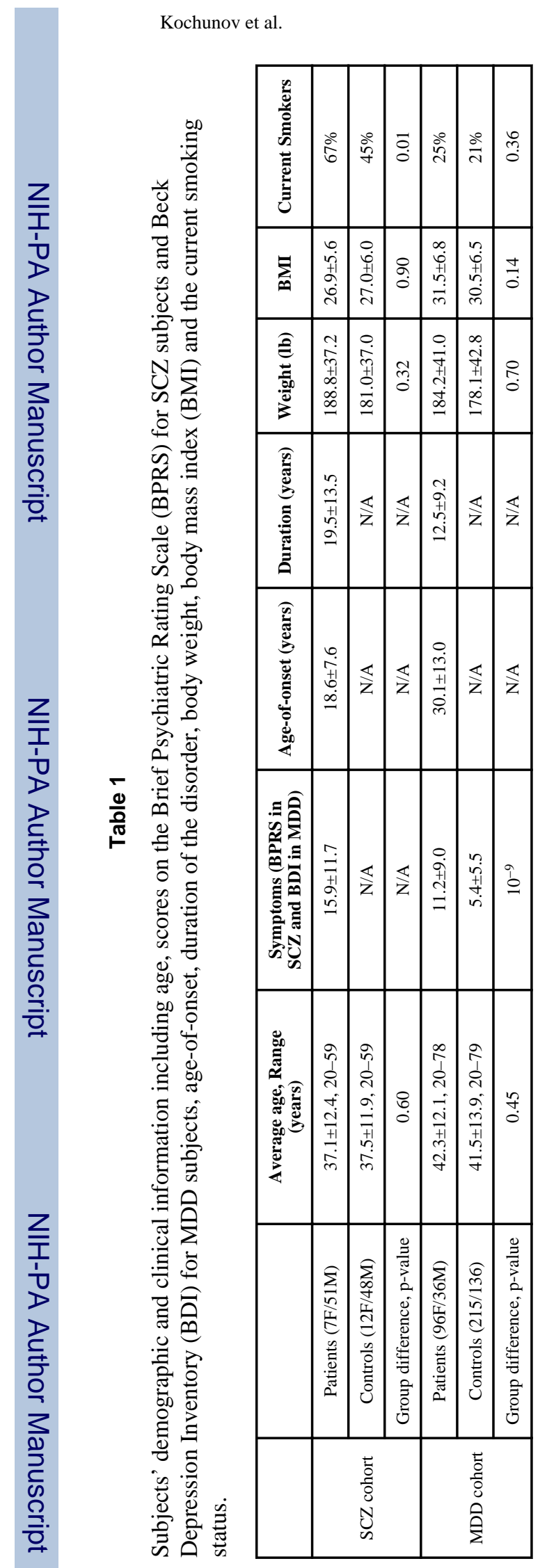

Biol Psychiatry. Author manuscript; available in PMC 2014 March 01. 


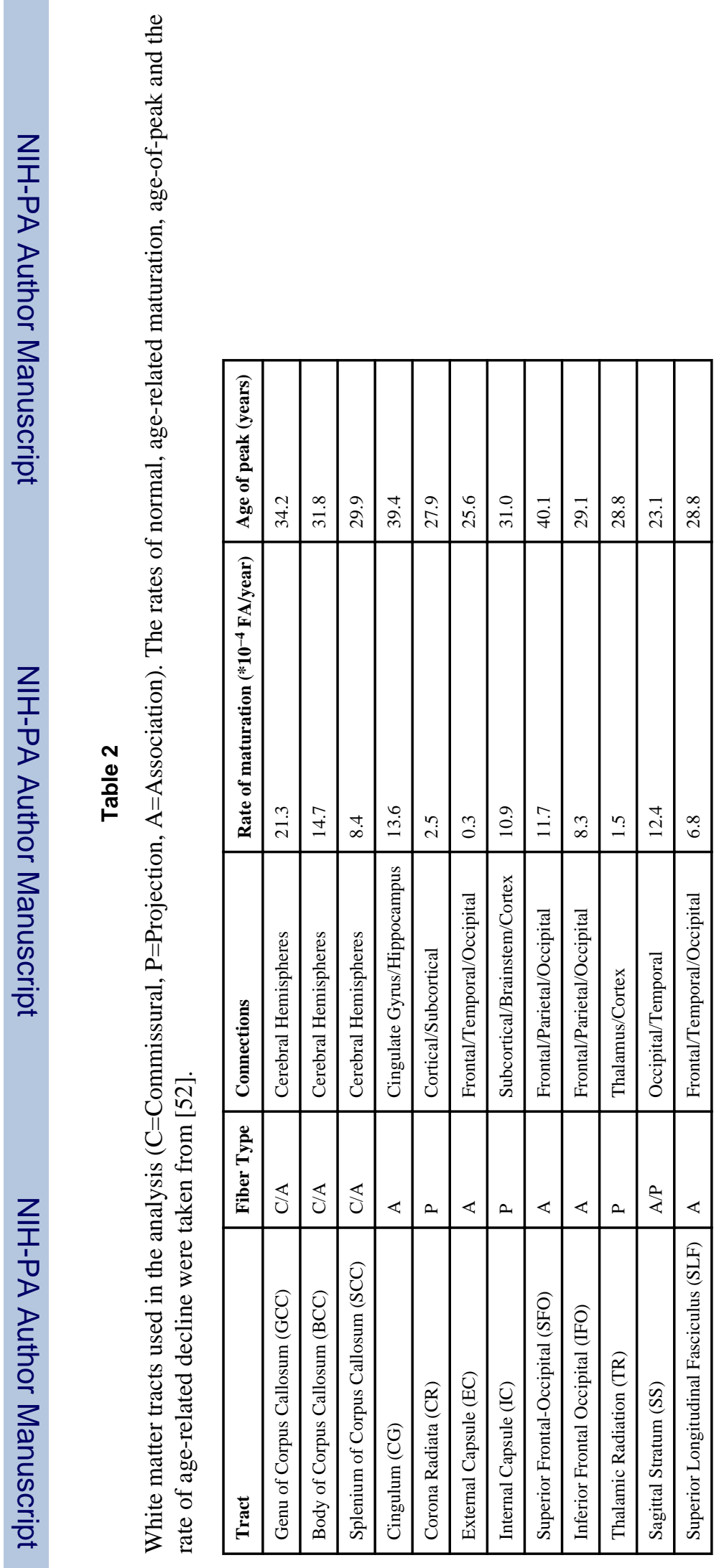




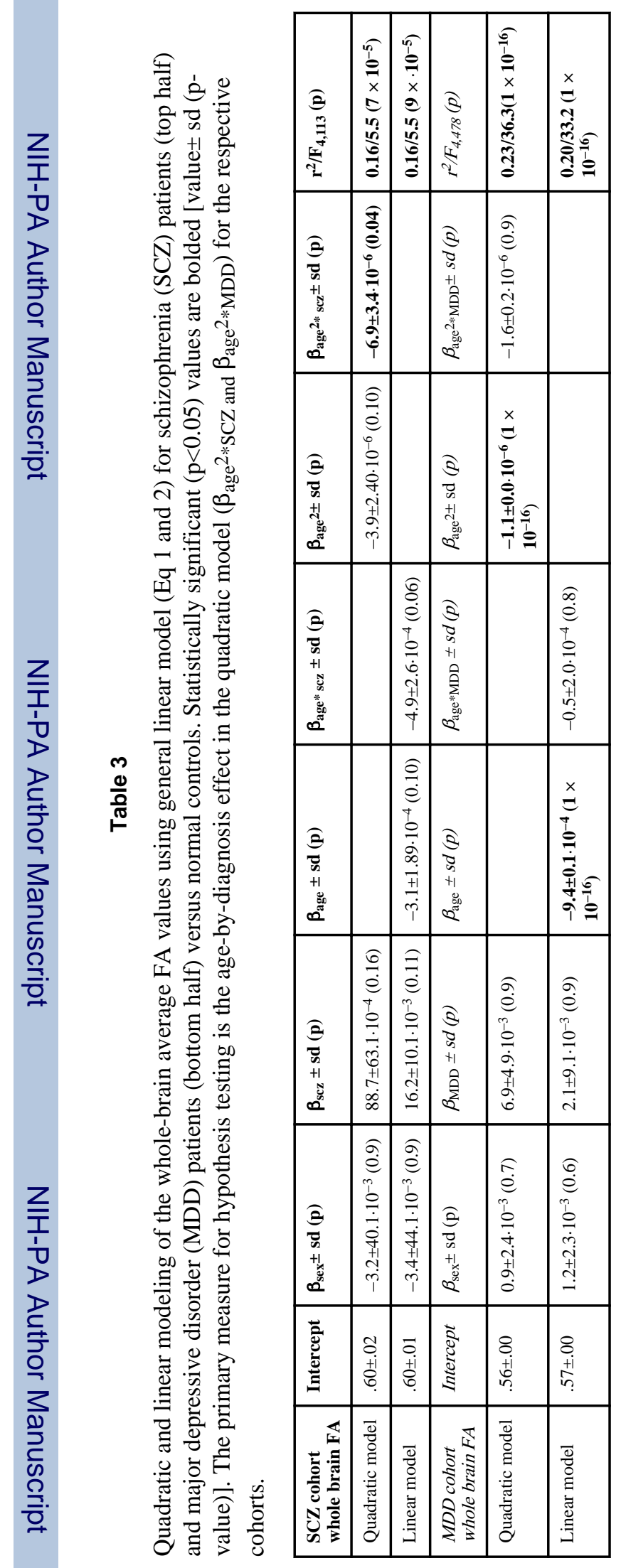

Biol Psychiatry. Author manuscript; available in PMC 2014 March 01. 


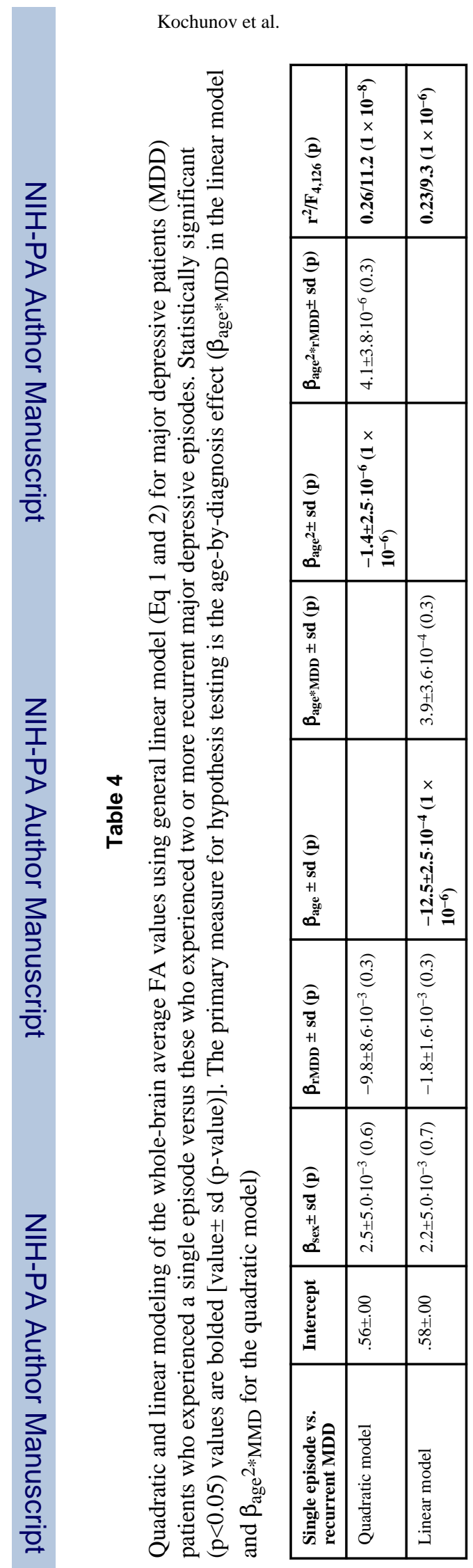

Biol Psychiatry. Author manuscript; available in PMC 2014 March 01. 